\title{
Design and Analysis of a Microstrip Antenna Based on Superconducting Material for Millimeter Wave Applications
}

\begin{abstract}
S. Djidel, M. Bouamar and D. Khedrouche*
LASS (Laboratoire d'Analyse des Signaux et Systèmes), Department of Electronics, University of M'sila, B.P. 166, Route Ichebilia, M'sila, 28000, Algeria

In this paper, we present a design and analysis of microstrip antenna based on superconducting material for millimeter wave applications. The proposed antenna is simulated using electromagnetic microwave studio computer simulator CST. The structure is made by two materials: the yttrium barium copper oxide (YBCO) thin film superconductor material as the patch and silicon Si as substrate with permittivity of 11.9. The superconductor material is characterized by a conductivity of $1.62 \times 10^{7} \mathrm{~S} / \mathrm{m}$ and critical high temperature of $89 \mathrm{~K}$. The simulated results concerning the return loss show that our structure has ultra wide band over 27 to $70 \mathrm{GHz}$. Low return loss of the superconductor antenna compared with his perfect conductor counterpart is achieved. In addition, good radiation pattern characteristics are obtained in the frequency band of operation. The proposed antenna has also overall size of $3 \times 3 \mathrm{~mm}^{2}$, which facilitate its integration in architecture of communications systems.
\end{abstract}

DOI: 10.12693/APhysPolA.131.109

PACS/topics: 74.78.-w, 74.72.-h, 84.40.Ba, 84.40.Ua, 84.40.Gf

\section{Introduction}

Nowadays, the need for higher data transmission rate $(\mathrm{Gb} / \mathrm{s})$ of wireless communication systems motivates the usage of millimeter wave frequency bands (e.g., 57$64 \mathrm{GHz}$ in USA, 59-66 GHz in Japan) [1]. Microstrip antennas of various shapes have recently received much attention and have been widely used in the range of millimeter-wave frequencies [2].

The discovery of new superconducting materials facilitates the development of microwave and millimeter-wave devices with better performance than conventional devices $[3,4]$. Advantages of using the superconducting materials at high frequencies are: very small losses which means low attenuation and low noise, smaller devices due to the lower losses, which leads to larger integration density, and the propagation time can be greatly reduced because of the smaller size and the shorter interconnects [5].

Superconducting materials have a great effect on the performance of microstrip antennas, due to the much lower surface resistance compared with normal conductor and high transition temperature over the boiling point of liquid nitrogen $[4,6]$. High $T_{c}\left(\mathrm{HT}_{c}\right)$ superconducting microstrip patch antennas have higher gain than their normal counterparts, but they suffer from the extremely narrow bandwidth, which severely limits their application [7].

In this paper, we design and analyse a new microstrip antenna structure based on superconducting material for millimeter wave application. The proposed antenna is simulated and performed using the microwave simulator

\footnotetext{
* corresponding author; e-mail:

djamel.khedrouche@univ-msila.dz
}

software studio computer CST. The superconductor material used is YBCO with conductivity $1.62 \times 10^{7} \mathrm{~S} / \mathrm{m}$ and $\mathrm{HT}_{c}$ of $89 \mathrm{~K}$. The simulation results concerning some characteristics such as the reflection coefficient (return loss) and the radiation pattern are discussed. In addition, a comparative study is presented to show the effect of superconducting material in these characteristics compared with a conventional microstrip antenna with a perfect conductor as a radiating patch.

\section{Antenna design}

The steps sequences of design antenna based on superconducting thin film material are shown in Fig. 1. The original antenna is depicted in Fig. 1a,b, it consists of a classical rectangular patch of dimensions $W_{p}=1.5 \mathrm{~mm}$ and $L_{p}=1.5 \mathrm{~mm}$. The patch is fed by a microstrip line, with width of $W_{f}=0.24 \mathrm{~mm}$ and length of $L_{f}=0.8 \mathrm{~mm}$, to achieve input impedance of $50 \Omega$ as shown in Fig. 1a. In Fig. 1b, we show a partial ground plane with length of $L_{g}=0.75 \mathrm{~mm}$ and the same width of the substrate $W_{s}=3 \mathrm{~mm}$. All these elements: the patch, the feeder, and the ground plane are made by YBCO superconducting thin film material with conductivity $1.62 \times 10^{7} \mathrm{~S} / \mathrm{m}$ and critical temperature $T_{c}=89 \mathrm{~K}$. The dielectric is made by silicon $(\mathrm{Si})$ with permittivity $\varepsilon_{r}=11.9$, length of $L_{s}=3 \mathrm{~mm}$ and having thickness of $H_{s}=0.154 \mathrm{~mm}$.

The proposed antenna, obtained by modifying the original antenna, is shown in Fig. 1c,d. The V-shaped patch has parametric dimensions of $W_{t}, W_{t 1}, L_{t}$ and $L_{t 1}$ as depicted in Fig. 1c. The dimensions $W_{t}, L_{t}$ and $L_{t 1}$ are set to $0.4 \mathrm{~mm}$ and $W_{t 1}$ to $0.3 \mathrm{~mm}$. For the purpose of impedance matching, a small rectangle is etched from the partial ground plane. The dimensions of this rectangle are $W_{c}$ and $L_{c}$ as indicated in Fig. $1 \mathrm{~d}$ and are set to $0.3 \mathrm{~mm}$ and $0.2 \mathrm{~mm}$, respectively. 

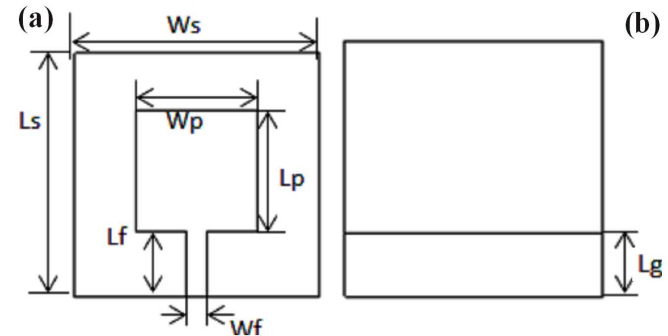

(c)

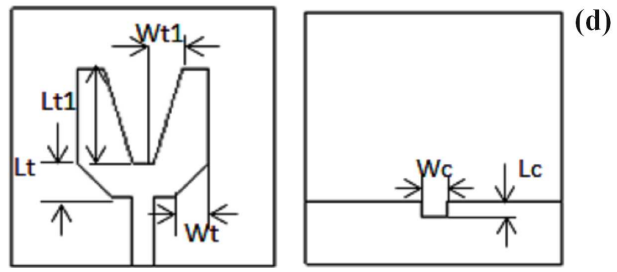

Fig. 1. Design sequences of proposed antenna: (a) top view, (b) bottom view, (c) top view, (d) bottom view.

\section{Results and discussion}

The simulated results are concerning the return loss coefficient $\left(S_{11}\right)$ and the radiation pattern, of the microstrip structure of Fig. 1, and are performed by CST commercial simulator. Figure 2 shows the comparison between the original and the proposed antenna in term of return loss. From the figure, we can observe that the original antenna had a discontinuity in the impedance bandwidth in the frequency band of interest for $\left(S_{11}<-10 \mathrm{~dB}\right)$ and have two bands (dual band). These bands are from 30 to $42 \mathrm{GHz}$ and from 72 to $77 \mathrm{GHz}$. To improve the bandwidth of this antenna, a modification on the patch is made to obtain the $\mathrm{V}$-shaped patch depicted in Fig. 1c. The corresponding return loss for this case is illustrated in Fig. 2. The curve (red line) demonstrate that our structure have an impedance bandwidth stretched from $27 \mathrm{GHz}$ to $70 \mathrm{GHz}$ for return loss $S_{11}<-10 \mathrm{~dB}$. In addition, it can be seen that the proposed antenna has two essential resonance frequencies. These frequencies are $30.5 \mathrm{GHz}$ and $65.78 \mathrm{GHz}$. The proposed antenna is well matched at these frequencies and shows a return loss of $-45 \mathrm{~dB}$ at $30.5 \mathrm{GHz}$ and $-40 \mathrm{~dB}$ at $65.78 \mathrm{GHz}$.

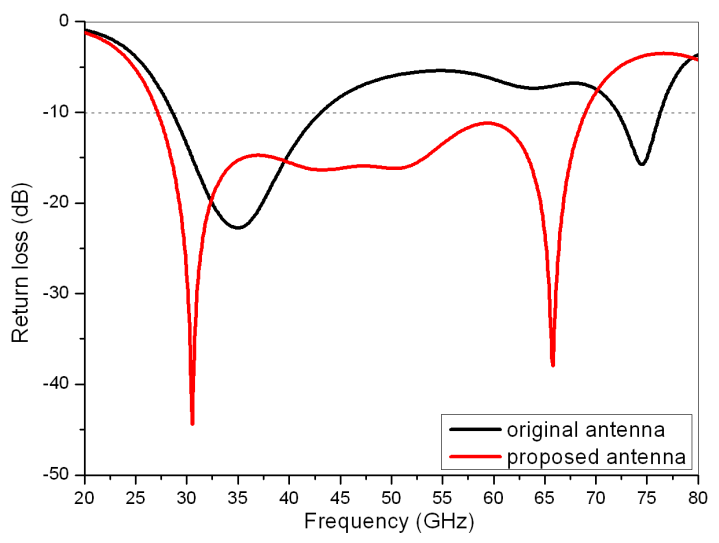

Fig. 2. Return loss of original and proposed antenna.

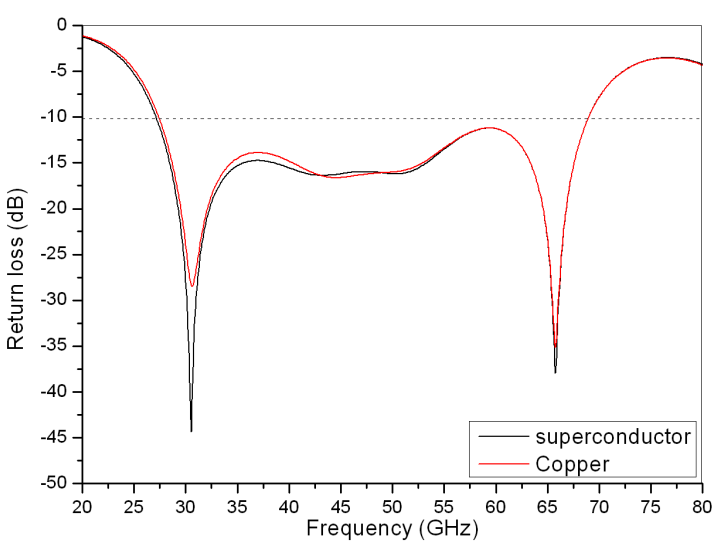

Fig. 3. Return loss of superconductor and perfect conductor patches.

In order to study the effect of superconductivity on the microstrip antenna performances, we plotted in Fig. 3 the reflection coefficient for a perfect conductor patch antenna (black line) and a superconducting patch antenna (red line), respectively. It can be seen that the return loss curves merge in the frequency band of interest. However, a more matching behavior is observed when we use the material YBCO compared with his copper counterpart, at the resonance frequency of $30.5 \mathrm{GHz}$, where we recorded a $-45 \mathrm{~dB}$ of return loss for superconductor material versus $-27 \mathrm{~dB}$ for the patch made by copper. This difference is due to the characteristic of the superconductor material which has much lower surface resistance compared with the perfect conductor. Thus, a better bandwidth matching is achieved using the thin film YBCO material.

The radiation pattern is a very important parameter in defining the performance of an antenna, because the role of an antenna is to radiate. The radiation pattern of the proposed antenna based on superconducting material is depicted in Figs. 4 and 5 at high resonance frequencies $30.5 \mathrm{GHz}$ and $65.78 \mathrm{GHz}$, respectively. The plots are performed in the $E$-plane for $\Phi=0^{\circ}$ and $H$-plane for $\Theta=90^{\circ}$. From these figures, we can see that the radiation pattern of the antenna have an omnidirectional behavior in the $E$-plane and a nearly omnidirectional
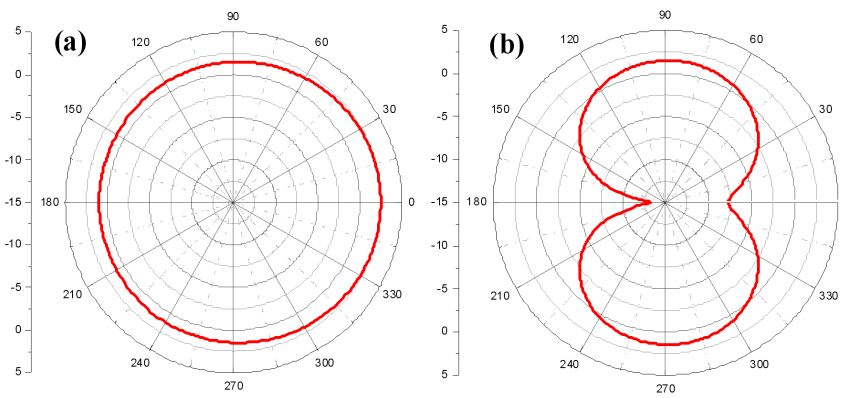

Fig. 4. Radiation pattern at $f=30.5 \mathrm{GHz}$ : (a) $E$ plane, (b) $H$-plane. 

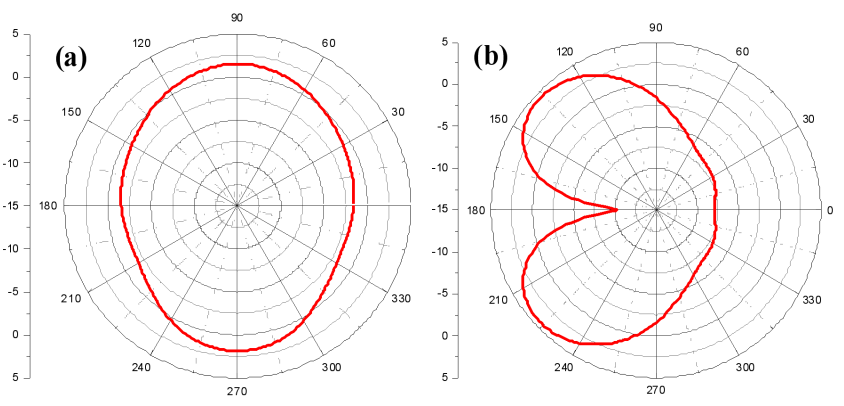

Fig. 5. Radiation pattern at $f=65.78 \mathrm{GHz}$ : (a) $E$ plane, (b) $H$-plane.

characteristic in the $H$-plane. We can observe also that the pattern is divided in two lobes in the $H$-plane at the resonance frequency of $65.78 \mathrm{GHz}$. This behavior is well known in radiation characteristics of microstrip antenna when the resonance frequency increases.

\section{Conclusion}

In this work, a design and analysis of a microstrip antenna based on superconducting material for millimeter wave applications is presented. The simulated results demonstrate that the proposed antenna has an ultra-wide band over 27 to up $70 \mathrm{GHz}$. We also showed that the performances of the proposed antenna using the superconductor material (YBCO) such as the return loss is enhanced compared with perfect conductor patch, especially at the resonance frequencies $30.5 \mathrm{GHz}$ and $65.78 \mathrm{GHz}$. The proposed antenna provides good monopole-like radiation patterns and it is miniature with a small size of $3 \times 3 \mathrm{~mm}^{2}$, which make it compatible with the integration technology of components in architecture of communications systems.

\section{References}

[1] X.J. Tang, S.Q. Xiao, B.Z. Wang, J. Wang, Int. J. Infrared Millim. Waves 28, 275 (2007).

[2] O. Barkat, A. Benghalia, Int. J. Infrared Millim. Waves 30, 1053 (2009).

[3] H.C.C. Fernandes, L.M. Caetano, H.M.C.A. Maia, J. Microwaves Optoelectron. Electromagn. Appl. 12, 269 (2013).

[4] S.F. Liu, X.W. Shi, Progr. Electromagn. Res. 77, 281 (2007).

[5] S. Bedra, T. Fortaki, A. Messai, R. Bedra, Wireless Pers. Commun. 86, 495 (2016).

[6] D. Khedrouche, A. Benghalia, J. Comput. Electron. 12, 297 (2013).

[7] S. Benkouda, M. Amir, T. Fortaki, A. Benghalia, J. Infrared Millim. Terahertz Waves 32, 1350 (2011). 